\title{
Comprehensive residency-based point-of-care ultrasound training program increases ultrasound utilization in the emergency department
}

\section{Wei-Lung Chen}

Cathay General Hospital

\section{Chan-Peng Hsu}

Hsinchu Cathay General Hospital

\section{Po-Han Wu}

Cathay General Hospital

Jiann-Hwa Chen

Cathay General Hospital

\section{Chien-Cheng Huang}

Chi Mei Medical Center

Jui-Yuan Chung ( $\sim$ bybarian@gmail.com )

Cathay General Hospital https://orcid.org/0000-0002-4823-555X

\section{Research article}

Keywords: point-of-care ultrasound, ultrasound utilization, ultrasound education, emergency department

Posted Date: May 12th, 2020

DOI: https://doi.org/10.21203/rs.3.rs-27350/v1

License: (c) (i) This work is licensed under a Creative Commons Attribution 4.0 International License. Read Full License

Version of Record: A version of this preprint was published at Medicine on February 5th, 2021. See the published version at https://doi.org/10.1097/MD.0000000000024644. 


\section{Abstract}

Background: Point-of-care ultrasonography (POCUS) is a prompt and simple tool for the urgent diagnosis and treatment of patients in the emergency department (ED). It has contributed to improvements in patient treatment quality, procedural safety, timeliness of care, diagnostic accuracy, and cost reduction. We developed a comprehensive residency-based POCUS training program for ED residents and determined its effect on ultrasound utilization in the ED.

Methods: We conducted a retrospective cohort study in the ED of a university-affiliated medical center, evaluating a centralized residency-based POCUS training course from July 2017 to June 2018 for ED residents that included 12 core ultrasound applications: trauma, intrauterine pregnancy, abdominal aortic aneurysm, echocardiography and hemodynamic assessment, hepatobiliary system, urinary tract, deep vein thrombosis, soft tissue and musculoskeletal, thoracic-airway, ocular, bowel, and procedural guidance. Each application comprised a combined lecture and hands-on practice session that lasted for 2 hours. Pre-tests and post-tests, including still image and video interpretation, were performed. Utilization of POCUS among ED residents before and after the POCUS training course (July 2016-June 2017 and July 2018-June 2019) was calculated and analyzed using the Wilcoxon signed-rank test.

Results: In total, 16 residents participated and completed the entire training course. The post-test score improved significantly compared to the pre-test score by a median of 12 points $(p=0.04)$. Utilization of POCUS among the ED residents increased significantly from 0.15 ultrasound studies per patient per year to 0.41 ultrasound studies per patient per year $(p<0.01)$ after completion of the entire training course. Increased POCUS scanning percentages over the cardiac, soft tissue, abdominal region, vascular system, procedural guidance, and ocular regions were also noted after providing the curriculum.

Conclusions: There was a significant increase in POCUS utilization among ED residents after a comprehensive residency-based POCUS training program.

\section{Background}

Point-of-care ultrasonography (POCUS) is an imaging technique performed by health care providers at the bedside, focusing on the patient's chief complaints, and interpreted in combination with their clinical manifestations. POCUS is especially essential in the crowded and fast-paced emergency department (ED) and is able to assist emergency medicine (EM) physicians in expediting prompt initial diagnosis, accurate decision making, and improving patient satisfaction.

The development of POCUS commenced in the 1990s, when the American College of Emergency Medicine (ACEP) published an article that supports the performance of POCUS in appropriately trained physicians [1]. This was further endorsed by the Society for Academic Emergency medicine in 1991 [2]. In 1994, a model curriculum for training physicians in emergency medicine ultrasonography was established by Mateer and colleagues [3]. Based on the positive influence of the use of POCUS in the ED, 
ultrasonography has been introduced as a standard part of the EM residency training program in the United States and Canada.

It was not until 2001 that the Accreditation Council for Graduate Medical Education (AGCME) mandated that all EM residents should become proficient in performing POCUS, and the ACEP also announced its very first emergency ultrasound guideline [4]. The 2001 POCUS guideline consists of seven ultrasound applications: trauma, pregnancy, abdominal aorta, cardiac, biliary, urinary tract, and procedural. These were expanded in 2016, with additional thoracic, bowel, deep vein thrombosis (DVT), ocular, and soft tissue/musculoskeletal, all together giving 12 core ultrasound applications [5].

The ED POCUS education in Taiwan was established by the Taiwan Society of Emergency Medicine (TSEM) according to the ACEP guidelines. Initially, the TSEM held multiple sessions of basic and advanced ultrasound workshops annually to promote POCUS among EM residents and physicians. The ability to perform emergency POCUS was included in the Taiwan EM milestone in 2016 by 15 EM education experts, according to the goal-directed focused ultrasound milestones consensus [6]. EM residents are mandated to attend both basic and advanced ultrasound workshops and perform at least 150 POCUS examinations before taking the board examination.

In order to follow the TSEM's EM POCUS education policy, we established an ED-centralized, comprehensive, and residency-based ultrasound training program. Instead of using subjective traditional clinical competence assessment tools, such as direct observation of procedural skills or mini-clinical evaluation exercise [7], we choose to evaluate the effect of the POCUS curriculum in a more objective way by comparing the amount of POCUS examination used by EM residents before and after the training program. To our knowledge, this is the first study to determine the effect of an ED POCUS education program by analyzing the utilization of ultrasonography.

\section{Methods}

\section{Study design, setting, and participants}

This study was conducted in a university-affiliated medical center, which includes 800 ward beds and 40 ED beds. Approximately 55,000 patients present to the ED annually. The ED includes 16 EM residents and

20 EM physicians. We commenced a comprehensive residency-based POCUS training program, according to the ACEP POCUS guideline, with 12 core applications from July 2017 to June 2018. Ultrasound utilization before (July 2016 to June 2017) and after (July 2018 to June 2019) the education program was retrospectively obtained and analyzed (Figure 1).

\section{Content of the POCUS education program}

The content of the POCUS education program was designed according to the ACEP POCUS guideline, including 12 core applications of trauma, pregnancy, abdominal aorta, cardiac, biliary, urinary tract, procedural, thoracic, bowel, DVT, ocular, and soft tissue/musculoskeletal [5]. All 16 EM residents were 
mandated to attend the training program, which was held monthly between July 2017 to June 2018. A combination of lectures and hands-on practice, each lasting for one hour, over a specific core application was arranged for each class. Well-designed pre- and post-tests were carried out before and after each lecture section. A detailed checklist was also established for each hands-on section.

\section{Equipment and faculty of the POCUS education program}

The ultrasound machine used for the education program was a Philips ClearVue 350, equipped with a curved array probe, phased array probe, and linear probe. Two emergency ultrasound experts were in charge of the training session. They were asked to reach a consensus before the education program and develop a unified lecture and hands-on content program for each month's class.

\section{Additional activities for the POCUS education program}

Additional activities, such as a mini POCUS game and POCUS diagnostic challenges, were conducted to enhance the EM residents' learning motivation. The mini POCUS game was held in the first half year of the training program (December 2018). The residents were split into groups of four and asked to accurately identify the sign or diagnosis on 20 POCUS images or videos. The POCUS diagnostic challenge was held in the second half year of the training session (June 2018). Two case scenarios were provided. The residents were split into four teams, and each team had to attempt to identify the final diagnosis of the two case scenarios via the POCUS protocol and techniques learned from the POCUS education program. The performance of each team was assessed using a thorough checklist. The teams

with the best performance in the mini POCUS game and POCUS diagnostic challenge were each awarded a certificate.

\section{Data collection and case and control group assignments}

Utilization of POCUS among EM residents was collected via the hospital information system as EM residents were obligated to prescribe a "POCUS examination" order whenever they performed POCUS. We could also obtain the POCUS image and video from each examination EM residents performed via the ultrasound machine's memory space. We then retrospectively collected the numbers of performed POCUS examinations by each EM resident before (control group, from July 2016 to June 2017) and after (case group, from July 2018 to June 2019) the training program. The pre-test and post-test scores of each EM resident were also collected and analyzed.

\section{Ethical statement}

This study was approved by the Institutional Review Board of the Cathay General Hospital and conducted according to the tenets of the Declaration of Helsinki. As the current study was an observational study, informed consent was not needed.

\section{Statistical analyses}


Statistical analyses were performed using SPSS 23.0 for Mac (IBM Corp., Chicago, IL, USA). Comparison of the pre-test and post-test results was analyzed using the Mann-Whitney $\mathrm{U}$ test and is displayed as a box plot. Utilization of POCUS by each EM resident was calculated as follows: number of performed ultrasound studies divided by the number of patients each resident saw in one year [8]. Ultrasound studies per patient per year before and after the training session were further analyzed using the MannWhitney $U$ test. The distribution of POCUS examinations by body region, including thoracic airway, echocardiography, soft tissue, abdominal region (trauma, pregnancy, biliary, and bowel), vascular system (aorta and deep vein thrombosis), urinary tract system, procedural guidance, and ocular and others, before and after the POCUS training program, is presented as a bar chart.

\section{Result}

Sixteen EM residents attended the entire POCUS training program between July 2017 and June 2018. The median lecture post-test score was significantly higher than the pre-test score $(70.0$, interquartile range [IQR]: 63.0-80.0; and 60.0, IQR: 48.0-70.0, respectively; $p=0.04$ ) (Figure 2). The average improvement in score was 10 points. The total numbers of patients seen by EM residents from July 2016 to June 2017 and from July 2018 to June 2019 were 21,227 and 28,122, respectively. The number of POCUS studies performed by the EM residents before and after the training program were 271 and 631, respectively. POCUS studies per patient per year among EM residents increased significantly after the training session from 0.16 (July 2016 to June 2017) to 0.41 (July 2018 to June 2019) $(p<0.01$ ) (Figure 3).

Distribution of POCUS examination by body region showed an increased proportion of POCUS examination in echocardiography ( $8.1 \%$ to $13.2 \%$ ), soft tissue regions ( $5.7 \%$ to $10.2 \%)$, abdominal regions (42.4\% to $47.0 \%)$, vascular system (5.6\% to $7.0 \%)$, and procedural guidance (2.3\% to $5.0 \%)$ after providing the POCUS training curriculum (Figure 4). The proportion of POCUS studies of the urinary tract system and thoracic-airway system decreased respectively from $30.2 \%$ to $12 \%$ and $5.6 \%$ to $5.4 \%$ after the training program.

\section{Discussion}

POCUS is an essential skill in the ED that could enhance the accuracy of diagnosis and decrease the time of patient disposition by integrating the results of POCUS examination and the patient's clinical information. It has also become an indispensable part of EM resident education such that several regions, including North America and Europe, have developed their own guidelines and regulations [9]. However, the impact of POCUS education in ultrasound utilization among EM residents has never been evaluated. Nicolay et al. studied the utilization of ultrasound in pediatric urology via ultrasound studies per patient per month [8]. Similarly, we designed the study by evaluating POCUS utilization in EM residents via ultrasound studies per patient per year. This method was more objective than directly comparing the total number of ultrasound studies performed by EM residents as the number of patients seen may vary over time and result in significant bias. 
A significant increase in the number of POCUS studies per patient per year performed by EM residents was noted after the comprehensive POCUS education program. This was further reinforced by the prominent improvement in the lecture post-tests scores, which indicate that EM residents obtained the necessary POCUS knowledge via the lecture section well. Due to the lack of practical experience, EM residents may be unassertive and hesitate to perform an actual ultrasound examination [10]. The handson section of the educational program may solve this problem by strengthening the EM residents' confidence and proficiency in performing POCUS in standardized patients. A study focusing on the impact of POCUS training on surgical residents' confidence also concluded that after a comprehensive POCUS lecture and hands-on curriculum, surgical residents had better self-efficacy and confidence levels regarding POCUS examination skills [11].

Academic competition played a crucial role in enhancing the learning motivation and knowledge regarding POCUS. An ultrasound competition for EM residents, called "SonoGames," was brought out by the Academy of Emergency Ultrasound during the annual SAEM conference in 2012 [12]. The game was so successful and popular that the number of participants doubled since its inauguration. The "Sound game," incorporated by Standford EM, which was an educational ultrasound event, also demonstrated prominent learning gains and overall satisfaction in the participants [13]. Likewise, we integrated such gamification into our POCUS training program and designed the mini POCUS game and POCUS diagnostic challenge to enhance EM residents' motivation in POCUS learning. After the competitions, we discovered that EM residents showed more enthusiasm for POCUS, leading to increased ultrasound utilization.

Effective POCUS education training program may benefit medical quality. Choi and colleagues discovered a prominent decrease in both ED patients' length of stay (LOS; 6.55 to 5.25 hours) and return visit (RV) rate $(6.4 \%$ to $5.25 \%)$ after a systematic POCUS education program [14]. A meta-analysis comparing the medical cost and radiation dosage between an ultrasound-computer tomography (CT) protocol and CTonly protocol for appendicitis evaluation showed lower medical costs for the limited ultrasound study at 88 United States Dollars (USD) per patient, whereas the CT imaging protocol was 547 USD per patient. Compared with the lack of radiation exposure in ultrasound examination, the radiation exposure for the CT protocol was approximately $12.4 \mathrm{mSv}$ and may lead to an excess of cancer deaths [15].

The distribution of POCUS examination by body regions before and after the POCUS training program may help us to improve our future education strategy. The lecture and hands-on sections will be reviewed and adjusted for the specific body system that has decreased POCUS utilization after the education program, for example, the urinary tract system and thoracic-airway system in this study. Decreased POCUS utilization in these two systems was probably due to the significant increase in POCUS usage in echocardiography, the soft tissue region, and abdominal region.

This study has some limitations, the first being its retrospective nature. Some key information, such as image quality, was unable to be measured and analyzed. Second, there was a rather small sample size with only 16 residents participating in the POCUS education program. Third, image quality of the POCUS 
images obtained by the EM residents was not assessed. Fourth, there may be missing case data as some EM residents may have forgotten to save the POCUS image. Fifth, individual bias may exist, as some EM residents were confident performing ultrasonography and may have over-performed POCUS, while some EM residents may have been unwilling to perform ultrasonography.

\section{Conclusion}

Compared to expensive, sophisticated, high-fidelity ultrasound simulators [16], a traditional lecture and hands-on ultrasound training program, in combination with academic competition, may be a more practical and cost-effective form of POCUS education. After a well-designed comprehensive residencybased POCUS training program, EM residents used ultrasound significantly more in the ED.

\section{Abbreviations}

POCUS: Point-of-care ultrasonography

ED: Emergency department

EM: Emergency medicine

ACEP: American College of Emergency Medicine

AGCME: Accreditation Council for Graduate Medical Education

DVT: Deep vein thrombosis

TSEM: Taiwan Society of Emergency Medicine

LOS: Length of stay

RV: Return visit

CT: Computer tomography

USD: United States Dollars

\section{Declaration}

\section{Ethics approval and consent to participate}

The study was approved by the Institutional Review Board of Cathay General Hospital. Because this was an observational study, the need for informed consent from patients was waived.

\section{Consent for publication}


Not applicable.

\section{Availability of data and materials}

The datasets supporting the conclusions of this article are available from the corresponding author on reasonable request.

\section{Competing interests}

All authors denied any conflict of interest.

\section{Funding}

This work was supported by Cathay General Hospital, [Grant number CGH-MR-B10902].

\section{Author contributions}

WLC, JYC, and CPH designed this study and wrote the manuscript. PHW performed the statistical analyses. JHC and $\mathrm{CCH}$ provided professional suggestions and wrote the manuscript. All authors read and approved the final manuscript.

\section{Acknowledgment}

We thank Editage for English language editing.

\section{References}

1. American College of Emergency Physicians: Council Resolution on Ultrasound. ACEP News, November 1990.

2. Society for Academic Emergency Medicine. Ultrasound Position Statement. SAEM Newsletter Summer 1991.

3. Hall M, DD P, Heller M, et al. Model curriculum for physician training in emergency ultrasonography. Ann Emerg Med 1994;23:95-102.

4. Hockberger RS, Binder LS, Graber MA, et al. The model of the clinical practice of emergency medicine. Ann Emerg Med 2001;37:745-70.

5. Ultrasound guidelines: emergency, point-of-care and clinical ultrasound guidelines in medicine. Ann Emerg Med 2017;69:e27-54.

6. Nelson M, Abdi A, Adhikari S, et al. Goal-directed focused ultrasound milestones revised: a multiorganizational consensus. Acad Emerg Med 2016;23:1274-9.

7. Pelgrim EA, Kramer AW, Mokkink HG, van den Elsen L, Grol RP, van der Vleuten CP. In-training assessment using direct observation of single-patient encounters: a literature review. Adv Health Sci Educ Theory Pract 2011;16:131-42. 
8. Nicolay L, Howe AS, Palmer LS. Ultrasound self-referral does not increase utilization in pediatric urology. Can J Urol 2017;24:9127-31.

9. Whitson MR, Mayo PH. Ultrasonography in the emergency department. Crit Care 2016;20:227. doi:10.1186/s13054-016-1399-x

10. Tahir B, Peterson DM, Heitkamp DE, et al. Taking resident feedback seriously: a hands-on ultrasound course. Acad Radiol 2016;23:1610-3. doi: 10.1016/j.acra.2016.08.020.

11. Kotagal M, Quiroga E, Ruffatto BJ, et al. Impact of point-of-care ultrasound training on surgical residents' confidence. J Surg Educ 2015;72:e82-7. doi: 10.1016/j.jsurg.2015.01.021.

12. Lewiss RE, Hayden GE, Murray A, et al. SonoGames: an innovative approach to emergency medicine resident ultrasound education. J Ultrasound Med 2014;33:1843-9.

13. Lobo V, Stromberg AQ, Rosston P. The sound games: introducing gamification into Stanford's orientation on emergency ultrasound. Cureus 2017;9:e1699. doi:10.7759/cureus.1699

14. Choi YJ, Jung JY, Kwon H. Effectiveness of education in point-of-care ultrasound-assisted physical examinations in an emergency department: a before-and-after study. Medicine (Baltimore) 2017;96:e7269. doi:10.1097/MD.0000000000007269

15. Parker L, Nazarian LN, Gingold EL, et al. Cost and radiation savings of partial substitution of ultrasound for CT in appendicitis evaluation: a national projection. AJR Am J Roentgenol 2014;202:124-35. doi: 10.2214/AJR.12.9642

16. Parks AR, Atkinson P, Verheul G, et al. Can medical learners achieve point-of-care ultrasound competency using a high-fidelity ultrasound simulator?: a pilot study. Crit Ultrasound J 2013;5:9. https://doi.org/10.1186/2036-7902-5-9

\section{Figures}




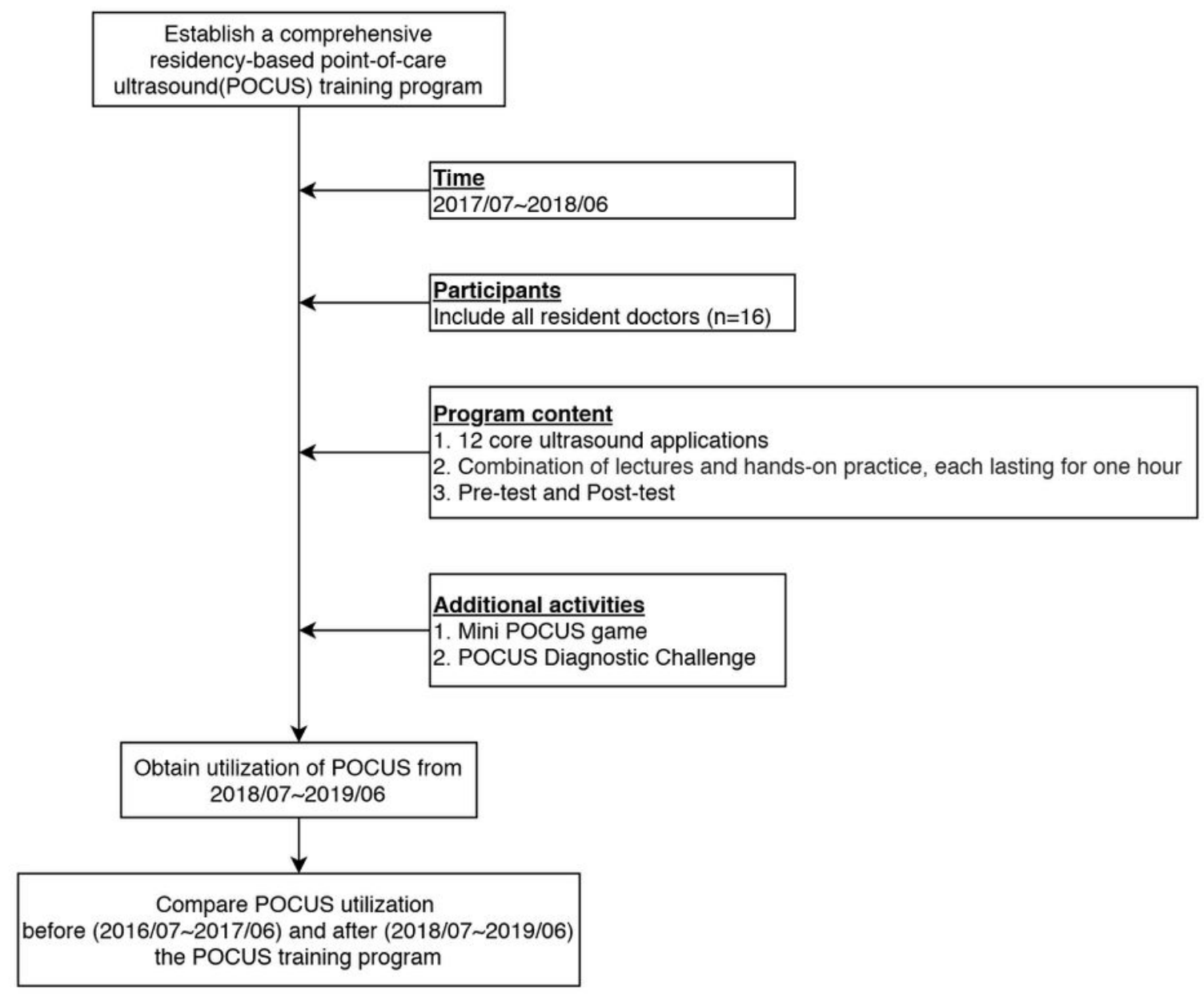

Figure 1

Flowchart of this study 


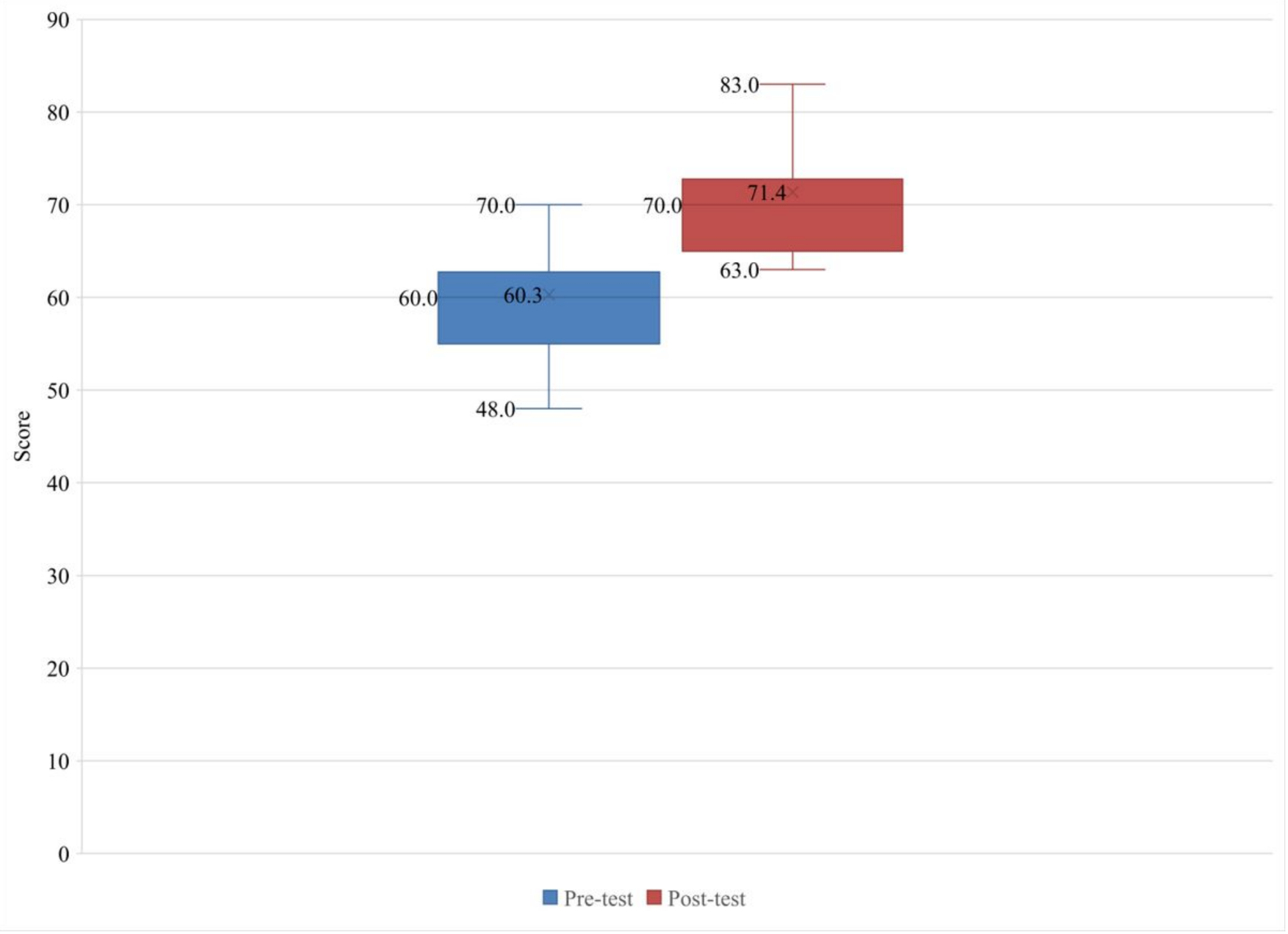

Figure 2

Box plot of pre-test and post-test scores 
0.45

0.41

0.40

$p<0.01$

0.35

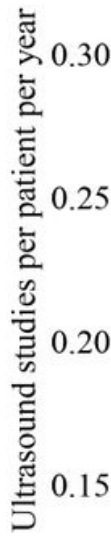

0.16

0.10

0.05

0.00

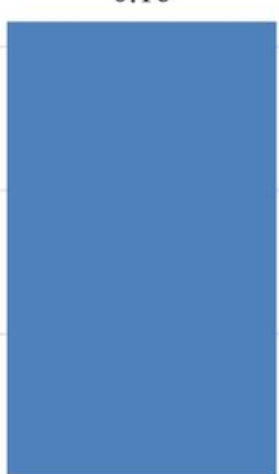

Before (2016/07-2017/06)

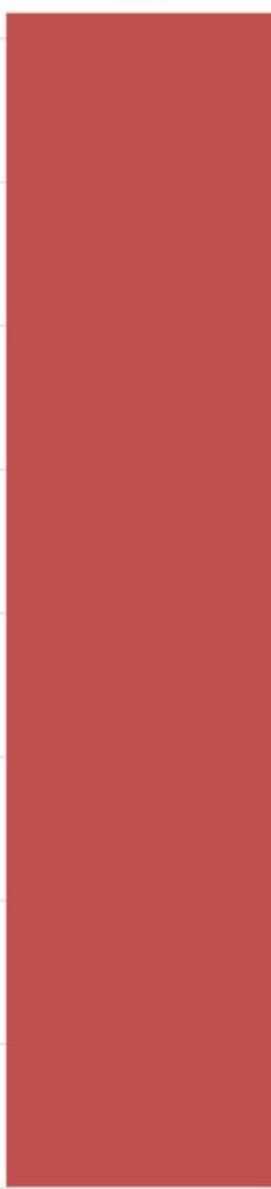

After (2018/07-2019/06)

Figure 3

Comparison of ultrasound studies per patient per year before (July 2016 to June 2017) and after (July 2018 to June 2019) the comprehensive POCUS education program. 


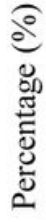

45

40

35

30

25

20

15

10

\begin{tabular}{|c|c|c|c|c|c|c|c|}
$\begin{array}{c}\text { Thoracic- } \\
\text { airway }\end{array}$ & $\begin{array}{c}\text { Echocardio } \\
\text { graphy }\end{array}$ & Soft tissue & $\begin{array}{c}\text { Abdomen } \\
\text { region }\end{array}$ & $\begin{array}{c}\text { Vascular } \\
\text { system }\end{array}$ & $\begin{array}{c}\text { Urinary } \\
\text { tract system }\end{array}$ & $\begin{array}{c}\text { Procedural } \\
\text { guidance }\end{array}$ & Ocular \\
\hline 5.6 & 8.1 & 5.7 & 42.4 & 5.6 & 30.2 & 2.3 & 0.1 \\
\hline 5.4 & 13.2 & 10.2 & 47 & 7 & 12 & 5 & 0.2 \\
\hline
\end{tabular}

Figure 4

Distribution of POCUS examination by body region before (July 2016 to June 2017) and after (July 2018 to June 2019) the comprehensive POCUS education program. 